\title{
Distributed finite-time leaderless consensus control for double-integrator multi-agent systems with external disturbances
}

\author{
Xiaoyan $\mathrm{He}^{\S}$, Qingyun Wang* \\ $\S$ Department of statistics and mathematics, Inner Mongolia University of Finance and \\ Economics, Huhhot, 010070, China \\ ${ }^{\star}$ Department of Dynamics and Control, Beihang University, Beijing, 100191, China
}

\begin{abstract}
We study the finite-time consensus control of double-integrator multi-agent systems with external bounded disturbances under the fixed leaderless network communication topology. Based on the relative position and the relative velocity information, a novel finite-time consensus protocol is constructed. By using the Lyapnuov finite-time theory, it is shown that the proposed protocol can coordinate the states of agents to guarantee the finite-time consensus under an undirected connected topology. And then we extend the results onto the strongly connected directed communication topology. Importantly, the finite convergence time of the leaderless systems is explicitly presented. Finally, the effectiveness of the results is illustrated by numerical simulation.
\end{abstract}

Keywords: Multi-agent system, Finite-time consensus control, Strongly connected topology, Double-integrator system

\section{Introduction}

In recent years, the study of consensus in multi-agent systems has been hot topic with application to the coordination of multi-agent systems, including formation flight of unmanned air vehicles(UAVs), clusters of satellites, self-

*Email: nmqingyun@163.com; Tel: +8610 82332003 
organization, automated highway systems, and congestion control in communication networks [1]. It is well known that the consensus of multi-agent systems have several advantages, including increased feasibility, accuracy, robustness, flexibility, cost, energy efficiency, and probability of success, etc. The objective of consensus problem is to design a proper distributed control algorithms such that a group of agents reach an agreement on some state of interest. Consensus has been investigated for multi-agent systems with various distributed control strategies, including adaptive control [2], sliding mode control [3, 4], Lyapunov control [5], optimal control [6], robust $H_{\infty}$ control [7], pining control [8, 9] etc.

Various control strategies have been studied for different multi-agent systems with various scenarios. According to the number of leaders existing in the systems, consensus can be classified into leaderless consensus, distributed tracking with one leader and containment control with multiple leaders. Recently, a variety of distributed consensus algorithms have been proposed to solve the leaderless consensus problems under different scenarios $[10,11,12,13]$. The leaderless consensus control problems have been investigated for first-order multi-agent systems in the presence of limited and unreliable information exchange with dynamically changing interaction topologies [10]. The leaderless consensus was studied for first-order nonlinear multi-agent systems under a directed communication network [11]. Typically, second-order consensus problem aims at studying how to make the agents move with the same velocity and converge to the same position. A new distributed observer-type consensus protocol has been proposed to study the second-order linear multi-agent systems with general linear node dynamics under the directed topology [12]. Wen et.al have investigated the consensus for a second-order integrator systems with a fixed directed topology and communication constraints, where each agent is assumed to share information only with its neighbors on some disconnected time intervals [13]. Some necessary and sufficient condition are presented to ensure the consensus of the second-order nonlinear systems [14, 15], where the steady state of velocities is time-invariant in undirected and directed topology networks.

It is noted that the above references mainly focus on the asymptotic consen- 
sus control for multi-agent systems. In fact, the convergence rate is a significant performance index for evaluating the effectiveness of a distributed consensus control of multi-agent systems. Compared with asymptotical consensus convergence, the finite-time convergence has faster convergence rates and higher robustness. Finite-time consensus for multi-agent systems with first-order and second-order dynamics was studied in $[16,17,18,19]$, respectively. Numerous researchers can improve the convergence rate by enlarging the coupling strength, optimizing the control gain or choosing better communication topology $[20,21,22,23]$. With the aid of sliding-mode control technique, distributed finite-time tracking controls for first-order nonlinear systems and second-order integrator systems have been investigated [24, 25], respectively. By using the sliding-mode control technique, containment control for second-order heterogeneous system and nonlinear systems with external disturbances has been studied $[26,27]$. By using the homogenous systems theory, the finite-time consensus tracking for a second-order nonlinear systems have been considered [28]. But, the precise time was not yet obtained. Meng et. al proposed distributed finite-time observers protocol for double integrator systems with a time-varying leader's velocity with bounded external disturbance [29].

To our knowledge, most results of the finite-time consensus control deal with the leader-following networks. However, there is no good result on the finitetime consensus problems of leaderless systems. Recently, a saturated protocol is proposed based on both relative position and relative velocity measurements for solving the finite-time consensus problem of second-order leaderless systems [30]. This is based on the homogenous theory, so the setting time is not present. Here, we will expand the existing some results $[13,14,15]$, the consensus are derived for second-order integrator systems and nonlinear systems by using the algebraic graph theory, matrix theory, and Lyapunov control approach. In particular, the distributed finite-time consensus control of the double-integrator leaderless systems with external bounded disturbances will be investigated in the undirected and directed communication topology networks. A novel consensus protocol is designed to coordinate the states of agents to converge to 
consensus in finite time under a connected undirected topology. And then, we will extend the results for the strongly connected directed topology. Compared with the existing results, one of the important innovation of this paper is that we extend the finite-time consensus problems from leader-following networks to leaderless systems, and the setting time of the leaderless system is explicitly presented.

The rest of this paper is organized as follows. Section 2 introduces some useful preliminaries of graph theory and the model description. In Section 3, the finite-time consensus control for second-order systems under the connected undirected graph is discussed, and we extend the results to the strongly connected communication graph. In Section 4, a numerical example is given to verify the theoretical analysis. Section 5 presents main conclusion.

\section{Preliminaries and model Description}

\subsection{Notations}

Let $R$ and $C$ be the sets of real and complex numbers, respectively. 1 and $\mathbf{0}$ represents the vector with all entries being one and the vector with all entries being zero, respectively. $I_{n}$ is the $n$-dimensional identity matrix. $A>0$ $(A<0)$ means that the matrix $A$ is positive (negative) definite. $\|A\|_{\infty}$ denotes the $\infty$-norm of matrix $A . \quad \lambda_{\min }(A)$ and $\lambda_{\max }(A)$ represents the minimal eigenvalue and the maximum eigenvalue of the matrix $A$, respectively. Given a vector $\xi=\left[\xi_{1}, \xi_{2}, \cdots, \xi_{p}\right]^{T}$ and constant $\kappa>0$, define $\operatorname{sig}(\xi)^{\kappa}=$ $\left[\operatorname{sign}\left(\xi_{1}\right)\left|\xi_{1}\right|^{\kappa}, \operatorname{sign}\left(\xi_{2}\right)\left|\xi_{2}\right|^{\kappa}, \cdots, \operatorname{sign}\left(\xi_{p}\right)\left|\xi_{p}\right|^{\kappa}\right]^{T}$ and $\operatorname{sign}(\xi)=\left[\operatorname{sign}\left(\xi_{1}\right), \operatorname{sign}\left(\xi_{2}\right)\right.$, $\left.\cdots, \operatorname{sign}\left(\xi_{p}\right)\right]^{T}$, where $\operatorname{sign}($.$) is the signum function. Let \operatorname{diag}\left(\xi_{1}, \xi_{2}, \cdots, \xi_{p}\right)$ represent a diagonal matrix with diagonal elements $\xi_{1}, \xi_{2}, \cdots, \xi_{p}$. The Kronecker product of matrices $A \in R^{m \times n}$ and $B \in R^{p \times q}$ is defined as

$$
A \otimes B=\left(\begin{array}{ccc}
a_{11} B & \cdots & a_{1 n} B \\
\vdots & \ddots & \vdots \\
a_{m 1} B & \cdots & a_{m n} B
\end{array}\right) .
$$


Lemma 1. ${ }^{[28]}$ Given matrices $A, B, C$ and $D$ with compatible dimensions, we have the following results,

(1) $(A \otimes B)(C \otimes D)=(A C) \otimes(B D)$,

(2) $(A \otimes B)+(A \otimes C)=A \otimes(B+C)$,

(3) $(A \otimes B)^{-1}=A^{-1} \otimes B^{-1},(A \otimes B)^{T}=A^{T} \otimes B^{T}$,

(4) if $A$ and $B$ are symmetric positive definite, so is $A \otimes B$.

Lemma 2. ${ }^{[29]}$ The following linear matrix inequality(LMI)

$$
\left(\begin{array}{cc}
M_{1}(x) & M_{2}(x) \\
M_{2}(x)^{T} & M_{3}(x)
\end{array}\right)>0,
$$

where $M_{1}(x)=M_{1}(x)^{T}, M_{3}(x)=M_{3}(x)^{T}$, is equivalent to one of the following conditions

1) $M_{1}(x)>0, M_{3}(x)-M_{2}(x)^{T} M_{1}(x)^{-1} M_{2}(x)>0$;

2) $M_{3}(x)>0, M_{1}(x)-M_{2}(x)^{T} M_{3}(x)^{-1} M_{2}(x)>0$.

\subsection{Graph theory notions}

For the leaderless multi-agent systems, we use graphs to represent the communication topology among $n$ agents. A topology graph $\mathcal{G}$ consists of a pair $(\mathcal{V}, \varepsilon)$, where $\mathcal{V}=\{1,2, \cdots, N\}$ and $\varepsilon \subseteq \mathcal{V} \times \mathcal{V}$ represent the set of nodes and the set of edges, respectively. A directed edge $(i, j) \in \varepsilon$ in graph $\mathcal{G}$ means that vehicle $j$ can obtain information from vehicle $i$, but not conversely. A undirected graph is defined such that $(j, i) \in \varepsilon$ implies $(i, j) \in \varepsilon$. A undirected graph $\mathcal{G}$ is said to be connected if there exists a path between each pair of distinct vertices. A directed graph is called strongly connected if there exists a directed path between each pair of distinct vertices. The adjacency matrix of a graph $\mathcal{G}$ is denoted by $\mathcal{A}=\left(a_{i j}\right) \in R^{n \times n}$, where $a_{i j}>0$ if $(j, i) \in \varepsilon$ and $a_{i j}=0$ otherwise. The in-degree of node $i$ is defined as $d_{i}=\sum_{i=1}^{n} a_{i j}$, and degree matrix is $\mathcal{D}=\operatorname{diag}\left\{d_{1}, d_{2}, \cdots, d_{n}\right\}$. The Laplacian matrix of the graph $\mathcal{G}$ associated with adjacency matrix $\mathcal{A}$ is designed as $\mathcal{L}=\left(l_{i j}\right)$, where $l_{i i}=\sum_{j=1, j \neq i}^{N} a_{i j}$ and $l_{i j}=-a_{i j}, i \neq j, i, j=1,2, \cdots, N$, i.e., $\mathcal{L}=\mathcal{D}-\mathcal{A}$. 


\subsection{Finite-time stability theory}

Here, we will give the result of the Lyapunov finite-time stability as discussed in [33].

Lemma 3. ${ }^{[30]}$ Consider the non-Lipschitz continuous nonlinear system $\dot{x}=f(x)$ whit $f(0)=0$. Suppose there are $C^{1}$ positive-definite function $V(x)$ defined on a neighborhood of the origin and real numbers $\gamma>0,(\beta>0)$ and $0<\alpha<1$, such that

1) $V(x)$ is positive definite,

2) $\dot{V}(x)+\gamma V^{\alpha} \leq 0$ (or $\left.\dot{V}(x)+\gamma V+\beta V^{\alpha} \leq 0\right)$, where $\dot{V}(x)=\frac{\partial V}{\partial x} f(x)$.

Then, the origin is a finite-time stable equilibrium, and the settling time depending on the initial state $x\left(t_{0}\right)=x_{0}$, satisfies

$$
T\left(x_{0}\right) \leq \frac{V^{1-\alpha}\left(x_{0}\right)}{\gamma(1-\alpha)}\left(\text { or } T\left(x_{0}\right) \leq \frac{1}{\gamma(1-\alpha)} \ln \frac{\gamma V^{1-\alpha}\left(x_{0}\right)+\beta}{\beta}\right),
$$

for all $x_{0}$ in some open neighborhood of the origin.

\subsection{Description of the second order multi-agent systems}

In this paper, we consider a multi-agent system with $n$ agents, whose dynamics are described by double-integrator dynamics as follows,

$$
\begin{gathered}
\dot{x}_{i}(t)=v_{i}(t), \\
\dot{v}_{i}(t)=u_{i}(t)+\delta_{i}(t), \quad i=1,2, \cdots, N,
\end{gathered}
$$

where $x_{i} \in R_{n}$ is the position, $v_{i} \in R_{n}$ is the velocity and $u_{i} \in R_{n}$ is the control input, $\delta_{i}$ is the unknown disturbance of the $i$ th agent. For further analysis, we assume that $\left\|\delta_{i}\right\| \leq \mathfrak{D}<\infty$. Denote $x=\left[x_{1}^{T}, x_{2}^{T}, \cdots, x_{N}^{T}\right]^{T}$, $v=\left[v_{1}^{T}, v_{2}^{T}, \cdots, v_{N}^{T}\right]^{T}, U=\left[u_{1}, u_{2}, \cdots, u_{N}\right]^{T}, \Delta=\left[\delta_{1}, \delta_{2}, \cdots, \delta_{N}\right]^{T}$.

Definition 1. ${ }^{[11]}$ The multi-agent system is said to achieve second-order finitetime consensus if for any initial conditions

$$
\lim _{t \rightarrow T}\left\|x_{i}-x_{j}\right\|=0, \quad \lim _{t \rightarrow T}\left\|v_{i}-v_{j}\right\|=0, \quad i, j=1,2, \cdots, n,
$$

where $T$ is a positive constant. 
The second-order consensus protocols in multi-agent dynamical systems have been described by Ren [10], Yu et. al [15] and Wen et. al [13], as follows,

$$
u_{i}=-\alpha \sum_{j=1}^{N} l_{i j} x_{j}-\beta \sum_{j=1}^{N} l_{i j} v_{j}, \quad i=1,2, \cdots, N .
$$

where $l_{i j}$ is the $(i, j)$ entry of the matrix $\mathcal{L}$, and $\alpha$ and $\beta$ represent the coupling strengths.

The control protocol (4) is commonly studied for the second-order consensus in the multi-agent systems. However, in some real situations, the finite-time consensus can be achieved by this. Based on the existing results, the objective of present paper is to design consensus control algorithms for systems (2) such that the finite-time consensus problem can be solved in finite time for the secondorder multi-agent systems for the undirected and directed network topologies. By means of the relative position and relative velocity information, a new finitetime consensus control algorithm is developed as follows,

$$
\begin{aligned}
& u_{i}=-\alpha\left[\sum_{j=1}^{N} l_{i j} x_{j}+\operatorname{sig}\left(\sum_{j=1}^{N} l_{i j} x_{j}\right)^{\tau}\right]-\beta\left[\sum_{j=1}^{N} l_{i j} v_{j}+\operatorname{sig}\left(\sum_{j=1}^{N} l_{i j} v_{j}\right)^{\tau}\right] \\
& -\mathfrak{D}\left[\operatorname{sign}\left(x_{i}-\sum_{j=1}^{N} x_{j}\right)+\operatorname{sign}\left(v_{i}-\sum_{j=1}^{N} v_{j}\right)\right], \quad i=1,2, \cdots, N .
\end{aligned}
$$

where $l_{i j}$ is the $(i, j)$ entry of the matrix $\mathcal{L}, \alpha$ and $\beta$ represent the coupling strengths, and $0<\tau<1$.

\section{Finite-time consensus analysis}

\subsection{Finite-time consensus control under undirected network topology}

In this subsection, we investigate the finite-time consensus for the secondorder multi-agent systems in the connected undirected network.

Assumpion 1. Assume the undirected communication topology between the agents is connected. 
Lemma 4. ${ }^{[13]}$ According to Assumption 1, the Laplacian matrix $\mathcal{L}$ has a simple eigenvalue 0 and all the other eigenvalues are positive. Moreover, the smallest nonzero eigenvalue $\lambda_{2}$ of $\mathcal{L}$ satisfies $\lambda_{2}=\min _{x \neq 0,1^{T} x=0} \frac{x^{T} \mathcal{L} x}{x^{T} x}$.

Let $\bar{x}_{i}=x_{i}-\frac{1}{N} \sum_{j=1}^{N} x_{j}, \bar{v}_{i}=v_{i}-\frac{1}{N} \sum_{j=1}^{N} v_{j}$ represent the average position and velocity of all agents in the system (2), respectively. So, by a simple calculation, we can get the error dynamical system as follows,

$$
\begin{gathered}
\dot{\bar{x}}_{i}=\bar{v}_{i}, \\
\dot{\bar{v}}_{i}=u_{i}-\frac{1}{N} \sum_{j=1}^{N} u_{j}+\delta_{i}-\frac{1}{N} \sum_{j=1}^{N} \delta_{j}, \quad i=1, \cdots, N .
\end{gathered}
$$

Let $\bar{x}=\left[\bar{x}_{1}^{T}, \bar{x}_{2}^{T}, \cdots, \bar{x}_{N}^{T}\right]^{T}=\left(M \otimes I_{n}\right) x, \bar{v}=\left[\bar{v}_{1}^{T}, \bar{v}_{2}^{T}, \cdots, \bar{v}_{N}^{T}\right]^{T}=\left(M \otimes I_{n}\right) v$, and $\bar{\delta}=\left[\bar{\delta}_{1}^{T}, \bar{\delta}_{2}^{T}, \cdots, \bar{\delta}_{N}^{T}\right]^{T}=\left(M \otimes I_{n}\right) \delta$, respectively, where $M=I-\frac{1}{N} \mathbf{1 1}^{T}$. Then, by substituting Eq.(5) into Eq.(6), the error system of the multi-agent system can be in a matrix form as

$$
\begin{aligned}
\dot{\bar{x}}= & \bar{v} \\
\dot{\bar{v}}= & -\alpha\left(M \otimes I_{n}\right)\left[\left(\mathcal{L} \otimes I_{n}\right) x+\operatorname{sig}\left[\left(\mathcal{L} \otimes I_{n}\right) x\right]^{\tau}\right] \\
& -\beta\left(M \otimes I_{n}\right)\left[\left(\mathcal{L} \otimes I_{n}\right) v+\operatorname{sig}\left[\left(\mathcal{L} \otimes I_{n}\right) v\right]^{\tau}\right] \\
& -\mathfrak{D}\left(M \otimes I_{n}\right)\left[\operatorname { s i g n } \left[\left(M \otimes I_{n}\right) x+\operatorname{sign}\left[\left(M \otimes I_{n}\right) v\right]+\left(M \otimes I_{n}\right) \Delta .(7)\right.\right.
\end{aligned}
$$

Noting the fact that $\mathcal{L} M=\mathcal{L}=M \mathcal{L}$, one has

$$
\begin{gathered}
\dot{\bar{x}}=\bar{v}, \\
\dot{\bar{v}}=-\alpha\left[\left(\mathcal{L} \otimes I_{n}\right) \bar{x}+\operatorname{sig}\left[\left(\mathcal{L} \otimes I_{n}\right) \bar{x}\right]^{\tau}\right]-\beta\left[\left(\mathcal{L} \otimes I_{n}\right) \bar{v}+\operatorname{sig}\left[\left(\mathcal{L} \otimes I_{n}\right) \bar{v}\right]^{\tau}\right] \\
-\mathfrak{D}\left(M \otimes I_{n}\right)[\operatorname{sign}(\bar{x})+\operatorname{sign}(\bar{v})]+\left(M \otimes I_{n}\right) \Delta .
\end{gathered}
$$

Let $\bar{\zeta}=\left(\bar{x}^{T}, \bar{v}^{T}\right)^{T}$, the system (8) can be written as

$$
\dot{\bar{\zeta}}=\left(B_{1} \otimes I_{n}\right) \bar{\zeta}+\left(B_{2} \otimes I_{n}\right) \operatorname{sig}(\overline{\mathcal{L}} \bar{\zeta})^{\tau}+\left(-\mathfrak{D} B_{3} \otimes I_{n}\right) \operatorname{sign}(\bar{\zeta})+\left(B_{3} \otimes I_{n}\right) \bar{\Delta}(9)
$$

where

$$
\begin{gathered}
\bar{\Delta}=\left\{\mathbf{0}^{T}, \Delta^{T}\right\}^{T}, \\
B_{1}=\left(\begin{array}{cc}
0_{N} & I_{N} \\
-\alpha \mathcal{L} & -\beta \mathcal{L}
\end{array}\right),
\end{gathered}
$$




$$
\begin{gathered}
B_{2}=\left(\begin{array}{cc}
0_{N} & 0_{N} \\
-\alpha I_{N} & -\beta I_{N}
\end{array}\right), \\
B_{3}=\left(\begin{array}{cc}
0_{N} & 0_{N} \\
-M & -M
\end{array}\right),
\end{gathered}
$$

and

$$
\overline{\mathcal{L}}=\left(\begin{array}{cc}
\mathcal{L}_{N} & 0_{N} \\
0_{N} & \mathcal{L}_{N}
\end{array}\right) .
$$

In what follows, the main results are given in detail.

Theorem 1. Suppose that the directed communication topology is strongly connected. Then, the finite-time consensus for the system(2) is achieved by the control protocol (5), if $\lambda_{2}>\alpha / \beta^{2}, 0<\tau<1$. More specifically, $x_{i} \rightarrow \frac{1}{N} \sum_{j=1}^{N} x_{j}$, $v_{i} \rightarrow \frac{1}{N} \sum_{j=1}^{N} v_{j}, i=1,2, \cdots, N$ in finite time.

Proof: According to the Lemma 4, the zero is a simple eigenvalue of $M$. In particular, $\mathbf{1}$ is a corresponding right eigenvector and one is the other eigenvalue with multiplicity $N-1$. Then, $\bar{x}=0$ and $\bar{v}=0$ if and only if $x_{1}=x_{2}=\cdots=x_{N}$ and $v_{1}=v_{2}=\cdots=v_{N}$, respectively. Therefore, the finite-time consensus problems can be solved if and only if $\bar{x}$ and $\bar{v}$ converge to zero in the finite time, respectively.

Construct the following Lyapunov function candidate:

$$
V_{1}(t)=\frac{1}{2} \bar{\zeta}^{T}\left(P_{1} \otimes I_{n}\right) \bar{\zeta}
$$

where

$$
P_{1}=\left(\begin{array}{cc}
\frac{\alpha \beta}{N}\left(\mathcal{L}+\mathcal{L}^{T}\right) & \frac{\alpha}{N} I_{N} \\
\frac{\alpha}{N} I_{N} & \frac{\beta}{N} I_{N}
\end{array}\right),
$$

It will be shown that $V_{1}(t)$ is a valid Lyapunov function for analyzing the error dynamics described by the error system (9). According to Lemma 1, we have

$$
\begin{aligned}
V_{1}(t)=\frac{\alpha \beta}{2 N} \bar{x}^{T}\left(\mathcal{L}+\mathcal{L}^{T}\right) \otimes & \left.I_{n}\right) \bar{x}+\frac{\alpha}{N} \bar{x}^{T}\left(I_{N} \otimes I_{n}\right) \bar{v}+\frac{\beta}{2 N} \bar{v}^{T}\left(I_{N} \otimes I_{n}\right) \bar{v} \\
& \geq \frac{1}{2} \bar{\zeta}^{T}\left(Q_{1} \otimes I_{n}\right) \bar{\zeta}
\end{aligned}
$$


where

$$
Q_{1}=\left(\begin{array}{cc}
\frac{\alpha \beta}{N} \lambda_{2}(\mathcal{L}) I_{N} & \frac{\alpha}{N} I_{N} \\
\frac{\alpha}{N} I_{N} & \frac{\beta}{N} I_{N}
\end{array}\right) .
$$

According to $\beta>0$ and $\lambda_{2}(\mathcal{L})>\alpha / 2 \beta^{2}$, Lemma 2 can be satisfied. Hence, we can get $Q_{1}>0$, then $V_{1}(t) \geq 0$, and $V_{1}(t)=0$ if and only if $\bar{\zeta}=\mathbf{0}_{2 N n}$. By using control protocol (5), we have the derivative of $V_{1}(t)$ as

$$
\begin{aligned}
& \dot{V}_{1}=\frac{1}{2} \bar{\zeta}^{T}\left[\left(P_{1} B_{1}+B_{1} P_{1}\right) \otimes I_{n}\right] \bar{\zeta}+\frac{1}{2} \bar{\zeta}^{T}\left[\left(P_{1} B_{2}+B_{2} P_{1}\right) \otimes I_{n}\right] \operatorname{sig}(\overline{\mathcal{L}} \bar{\zeta})^{\tau} \\
& +\frac{1}{2} \bar{\zeta}^{T}\left[-\mathfrak{D}\left(P_{1} B_{3}+B_{3} P_{1}\right) \otimes I_{n}\right] \operatorname{sign}(\bar{\zeta})+\frac{1}{2} \bar{\zeta}^{T}\left[\left(P_{1} B_{3}+B_{3} P_{1}\right) \otimes I_{n}\right] \bar{\Delta} \\
& =\frac{1}{2} \bar{\zeta}^{T}\left[\left(\begin{array}{cc}
\frac{-\alpha^{2}}{N}\left(\mathcal{L}+\mathcal{L}^{T}\right) & 0_{N} \\
0_{N} & \frac{-\beta^{2}}{N}\left(\mathcal{L}+\mathcal{L}^{T}\right)+\frac{2 \alpha}{N} I_{N}
\end{array}\right) \otimes I_{n}\right] \bar{\zeta} \\
& +\frac{1}{2} \bar{\zeta}^{T}\left[\left(\begin{array}{cc}
-\frac{\alpha^{2}}{N} I_{N} & -\frac{\alpha \beta}{N} I_{N} \\
-\frac{\alpha \beta}{N} I_{N} & -\frac{\beta^{2}}{N} I_{N}
\end{array}\right) \otimes I_{n}\right] \operatorname{sig}(\overline{\mathcal{L}} \bar{\zeta})^{\tau} \\
& +\frac{1}{2} \bar{\zeta}^{T}\left[\left(\begin{array}{cc}
-\frac{\alpha}{N} \mathfrak{D} M & -\frac{\alpha}{N} \mathfrak{D} M \\
-\left(\frac{\alpha \beta}{N} \mathfrak{D} M+\frac{\alpha+\beta}{N} \mathfrak{D} M\right) & -\frac{\alpha+2 \beta}{N} \mathfrak{D} M
\end{array}\right) \otimes I_{n}\right] \operatorname{sign}(\bar{\zeta}) \\
& +\frac{1}{2} \bar{\zeta}^{T}\left[\left(\begin{array}{cc}
\frac{\alpha}{N} M & \frac{\alpha}{N} M \\
\left(\frac{\alpha \beta}{N} M+\frac{\alpha+\beta}{N} M\right) & \frac{\alpha+2 \beta}{N} M
\end{array}\right) \otimes I_{n}\right] \bar{\Delta} \\
& =\frac{1}{2} \bar{\zeta}^{T}\left[\left(\begin{array}{cc}
\frac{-\alpha^{2}}{N}\left(\mathcal{L}+\mathcal{L}^{T}\right) & 0_{N} \\
0_{N} & \frac{-\beta^{2}}{N}\left(\mathcal{L}+\mathcal{L}^{T}\right)+\frac{2 \alpha}{N} I_{N}
\end{array}\right) \otimes I_{n}\right] \bar{\zeta} \\
& +\frac{1}{2} \bar{\zeta}^{T}\left[\left(\begin{array}{cc}
-\frac{\alpha^{2}}{N} & -\frac{\alpha \beta}{N} \\
-\frac{\alpha \beta}{N} & -\frac{\beta^{2}}{N}
\end{array}\right) \otimes I_{n N}\right] \operatorname{sig}(\overline{\mathcal{L}} \bar{\zeta})^{\tau} \\
& +\frac{1}{2} \bar{\zeta}^{T}\left[-\mathfrak{D}\left(\begin{array}{cc}
\frac{\alpha}{N} & \frac{\alpha}{N} \\
\left(\frac{\alpha \beta}{N}+\frac{\alpha+\beta}{N}\right) & \frac{\alpha+2 \beta}{N}
\end{array}\right) \otimes M \otimes I_{n}\right] \operatorname{sign}(\bar{\zeta}) \\
& +\frac{1}{2} \bar{\zeta}^{T}\left[\left(\begin{array}{cc}
\frac{\alpha}{N} & \frac{\alpha}{N} \\
\left(\frac{\alpha \beta}{N}+\frac{\alpha+\beta}{N} M\right) & \frac{\alpha+2 \beta}{N}
\end{array}\right) \otimes M \otimes I_{n}\right] \bar{\Delta} \\
& \leq-\bar{\zeta}^{T}\left(P_{2} \otimes I_{n N}\right) \bar{\zeta}-\bar{\zeta}^{T}\left(P_{3} \otimes I_{n N}\right) \overline{\mathcal{L}}^{\tau} \operatorname{sig}(\zeta)^{\tau} \\
& -\frac{1}{2} \mathfrak{D} \bar{\zeta}^{T}\left(P_{4} \otimes M \otimes I_{n}\right) \operatorname{sign}(\bar{\zeta})+\frac{1}{2} \bar{\zeta}^{T}\left(P_{3} \otimes M \otimes I_{n}\right) \bar{\Delta} \\
& =-\bar{\zeta}^{T}\left(P_{2} \otimes I_{n N}\right) \bar{\zeta}-\bar{\zeta}^{T}\left(P_{3} \otimes I_{n N}\right) \overline{\mathcal{L}}^{\tau} \operatorname{sig}(\zeta)^{\tau} \\
& -\frac{1}{2} \bar{\zeta}^{T}\left(P_{3} \otimes M \otimes I_{n}\right)[\mathfrak{D} \operatorname{sign}(\bar{\zeta})-\bar{\Delta}] \text {. }
\end{aligned}
$$


Using the assumption that $\left\|\delta_{i}\right\| \leq \mathfrak{D}<\infty$, we have

$$
\dot{V}_{1} \leq-\bar{\zeta}^{T}\left(P_{2} \otimes I_{n N}\right) \bar{\zeta}-\bar{\zeta}^{T}\left(P_{3} \otimes I_{n N}\right) \overline{\mathcal{L}}^{\tau} \operatorname{sig}(\zeta)^{\tau}
$$

where

$$
\begin{gathered}
P_{2}=\left(\begin{array}{cc}
\alpha^{2} \lambda_{2}(\mathcal{L}) & 0 \\
0 & \beta^{2} \lambda_{2}(\mathcal{L})-\alpha
\end{array}\right), \\
P_{3}=\left(\begin{array}{cc}
-\frac{\alpha^{2}}{2 N} & -\frac{\alpha \beta}{2 N} \\
-\frac{\alpha \beta}{2 N} & -\frac{\beta^{2}}{2 N}
\end{array}\right)
\end{gathered}
$$

and

$$
P_{4}=\left(\begin{array}{cc}
\frac{\alpha}{N} & \frac{\alpha}{N} \\
\frac{\alpha \beta}{N}+\frac{\alpha+\beta}{N} M & \frac{\alpha+2 \beta}{N}
\end{array}\right) .
$$

Then, we have

$$
\begin{gathered}
V_{1}(t)=\frac{1}{2} \bar{\zeta}^{T}\left(P_{1} \otimes I_{n}\right) \bar{\zeta} \\
=\frac{\alpha \beta}{2 N} \bar{x}^{T}\left[\left(\mathcal{L}+\mathcal{L}^{T}\right) \otimes I_{n}\right] \bar{x}+\frac{\alpha}{N} \bar{x}^{T}\left(I_{N} \otimes I_{n}\right) \bar{v}+\frac{\beta}{2 N} \bar{v}^{T}\left(I_{N} \otimes I_{n}\right) \bar{v}, \\
\leq \frac{\alpha \beta}{N} \lambda_{2}(\mathcal{L}) \bar{x}^{T}\left(I_{N} \otimes I_{n}\right) \bar{x}+\frac{\alpha}{N} \bar{x}^{T}\left(I_{N} \otimes I_{n}\right) \bar{v}+\frac{\beta}{2 N} \bar{v}^{T}\left(I_{N} \otimes I_{n}\right) \bar{v}, \\
\leq \bar{\zeta}^{T}\left(P_{5} \otimes I_{N n}\right) \bar{\zeta},
\end{gathered}
$$

where the positive matrix

$$
P_{5}=\left(\begin{array}{cc}
\frac{\alpha \beta}{2 N} & \frac{\alpha}{2 N} \\
\frac{\alpha}{2 N} & \frac{\beta}{2 N}
\end{array}\right) .
$$

Thus, according to Eq.(14), we can get the following inequality

$$
V_{1}(t) \leq \lambda_{\max }\left(P_{5}\right) \bar{\zeta}^{T} \bar{\zeta}=m_{3} \bar{\zeta}^{T} \bar{\zeta},
$$

where $m_{3}=2 \alpha \beta \lambda_{2}(\mathcal{L})+\frac{\sqrt{\left(2 \alpha \beta \lambda_{2}(\mathcal{L})-\beta\right)^{2}+4 \alpha^{2}}}{4}$.

And according to Eq.(13), we can get the following inequality

$$
\begin{gathered}
\dot{V}_{1}(t) \leq-\lambda_{\min }\left(P_{2}\right) \bar{\zeta}^{T} \bar{\zeta}-\lambda_{\min }\left(P_{3}\right) \lambda_{2}(\mathcal{L})^{\tau} \bar{\zeta}^{T} \operatorname{sig}(\bar{\zeta})^{\tau} \\
=-m_{1} \bar{\zeta}^{T} \bar{\zeta}-m_{2} \bar{\zeta}^{T} \operatorname{sig}(\bar{\zeta})^{\tau},
\end{gathered}
$$

where $m_{1}=\min \left\{\frac{\alpha^{2} \lambda_{2}(\mathcal{L})}{N}, \frac{\beta^{2} \lambda_{2}(\mathcal{L})-\alpha}{N}\right\}, m_{2}=\frac{\alpha^{2}+\beta^{2}}{2 N} \lambda_{2}^{\tau}(\mathcal{L})$. Combining Eq.(15) with Eq.(16), we have

$$
\dot{V}_{1}(t) \leq-\frac{m_{1}}{m_{3}} V_{1}(t)-\frac{m_{2}}{m_{3}} V_{1}(t)^{\frac{1+\tau}{2}} .
$$


According to Lemma 3 and inequality (17), we can get the setting time

$$
T=\frac{2 m_{3}}{m_{1}(1-\tau)} \ln \frac{m_{1} V^{1-\tau}(0)+m_{2}}{m_{2}} .
$$

Hence $x_{i} \rightarrow \frac{1}{N} \sum_{j=1}^{N} x_{j}, v_{i} \rightarrow \frac{1}{N} \sum_{j=1}^{N} v_{j}, i=1,2, \cdots, N$ in finite-time.

\subsection{Finite-time consensus control under directed network topology}

In the last subsection, we assume that the graph is undirected. In fact, the corresponding results can be extended to the directed graph, as shown in the following discussion.

Definition 2. ${ }^{[28]}$ The Laplacian matrix $\mathcal{L}$ of the directed network $\mathcal{G}$ is reducible if there is a permutation matrix $M \in \mathrm{R}^{n \times n}$ and an integer $m, 1 \leq m \leq n-1$, such that

$$
M^{T} \mathcal{L} M=\left(\begin{array}{cc}
\widetilde{M}_{11} & 0 \\
\widetilde{M}_{21} & \widetilde{M}_{22}
\end{array}\right) .
$$

where $\widetilde{M}_{11} \in \mathrm{R}^{m \times m}, \widetilde{M}_{21} \in \mathrm{R}^{(m-n) \times m}$ and $\widetilde{M}_{22} \in \mathrm{R}^{(n-m) \times(n-m)}$. Otherwise, the Laplacian matrix $\mathcal{L}$ is called irreducible.

Assumpion 2. The directed communication topology $\mathcal{G}$ between the agents of the systems (2) is strongly connected.

Lemma 5. ${ }^{[31]}$ Under Assumption 2, its Laplacian matrix $\mathcal{L}$ is irreducible and satisfies $\mathcal{L} \mathbf{1}=0$. Furthermore, there exists a positive vector $\xi=\left(\xi_{1}, \xi_{2}, \cdots, \xi_{n}\right)^{\mathrm{T}}$ such that $\xi^{\mathrm{T}} \mathcal{L}=0$. In addition, $\widehat{\mathcal{L}}=\left(\frac{1}{2}\right)\left(\Xi \mathcal{L}+\mathcal{L}^{T} \Xi\right)$ is a symmetric matrix, where $\Xi=\operatorname{diag}\left(\xi_{1}, \xi_{2}, \cdots, \xi_{n}\right)^{\mathrm{T}}$ and $\sum_{j=1}^{N} \mathcal{L}_{i j}=\sum_{j=1}^{N} \mathcal{L}_{j i}=0$ for all $i, j=$ $1,2, \cdots, N$.

Definition 3. ${ }^{[31]}$ For a strongly connected network with Laplacian matrix $\mathcal{L}$, the general algebraic connectivity is defined by

$$
a(\mathcal{L})=\min _{x^{T} \xi=0, x \neq 0} \frac{x^{T} \widehat{\mathcal{L}} x}{x^{T} \Xi x},
$$

and let

$$
b(\mathcal{L})=\max _{x^{T} \xi=0, x \neq 0} \frac{x^{T} \widehat{\mathcal{L}} x}{x^{T} \Xi x}
$$


where $\widehat{\mathcal{L}}=\left(\Xi \mathcal{L}+\mathcal{L}^{T} \Xi\right) / 2, \Xi=\operatorname{diag}\left(\xi_{1}, \xi_{2}, \cdots, \xi_{n}\right)^{\mathrm{T}}, \xi=\left(\xi_{1}, \xi_{2}, \cdots, \xi_{n}\right)^{\mathrm{T}}>0$, and $\Xi^{T} \mathcal{L}=0, \sum_{j=1}^{N} \xi_{i}=1$.

Let $\bar{x}_{i}^{d}=x_{i}-\sum_{j=1}^{N} \xi_{j} x_{j}, \bar{v}_{i}^{d}=v_{i}-\sum_{j=1}^{N} \xi_{j} v_{j}$ represent the position and velocity vectors relative to the weighted average position and velocity vectors of the agents in system (2), respectively. And $\xi=\left(\xi_{1}, \xi_{2}, \cdots, \xi_{N}\right)^{N}$ is the positive left eigenvector of Laplacian matrix $\mathcal{L}$ associated with its zero eigenvalue, satisfying $\xi^{T} \mathbf{1}_{N}=1$. So we do a simple calculation to get the error dynamical system as follows,

$$
\begin{gathered}
\dot{\bar{x}}_{i}^{d}=\bar{v}_{i}^{d}, \\
\dot{\bar{v}}_{i}^{d}=u_{i}-\sum_{j=1}^{N} \xi_{j} u_{j}+\delta_{i}-\sum_{j=1}^{N} \xi_{j} \delta_{j}, \quad i=1, \cdots, N,
\end{gathered}
$$

Let $\bar{x}_{d}=\left[\left(\bar{x}_{1}^{d}\right)^{T},\left(\bar{x}_{2}^{d}\right)^{T}, \cdots,\left(\bar{x}_{N}^{d}\right)^{T}\right]^{T}=\left(M_{d} \otimes I_{n}\right) x$ and $\bar{v}_{d}=\left[\left(\bar{v}_{1}^{d}\right)^{T},\left(\bar{v}_{2}^{d}\right)^{T}, \cdots\right.$, $\left.\left(\bar{v}_{N}^{d}\right)^{T}\right]^{T}=\left(M_{d} \otimes I_{n}\right) v$, respectively, where $M_{d}=I-\mathbf{1} \xi^{T}$. Then, by substituting Eq.(5) into Eq.(20), the error system of the multi-agent system can be in a matrix form as

$$
\begin{gathered}
\dot{\bar{x}}_{d}=\bar{v}_{d}, \\
\dot{\bar{v}}_{d}=-\alpha\left[\left(M_{d} \otimes I_{n}\right)\left(\left(\mathcal{L} \otimes I_{n}\right) x+\operatorname{sig}\left[\left(\mathcal{L} \otimes I_{n}\right) x\right]^{\tau}\right)\right] \\
-\beta\left[\left(M_{d} \otimes I_{n}\right)\left(\left(\mathcal{L} \otimes I_{n}\right) v+\operatorname{sig}\left[\left(\mathcal{L} \otimes I_{n}\right) v\right]^{\tau}\right)\right] \\
-\mathfrak{D}\left(M_{d} \otimes I_{n}\right)\left[\operatorname { s i g n } \left[\left(\mathcal{L} \otimes I_{n}\right) x+\operatorname{sign}\left[\left(\mathcal{L} \otimes I_{n}\right) v\right]+\left(M_{d} \otimes I_{n}\right) \Delta .\right.\right.
\end{gathered}
$$

Noting the fact that $\mathcal{L} M_{d}=\mathcal{L}=M_{d} \mathcal{L}$, one has

$$
\begin{gathered}
\dot{\bar{x}}_{d}=\bar{v}_{d}, \\
\dot{\bar{v}}_{d}=-\alpha\left[\left(\mathcal{L} \otimes I_{n}\right) \bar{x}_{d}+\operatorname{sig}\left[\left(\mathcal{L} \otimes I_{n}\right) \bar{x}_{d}\right]^{\tau}\right] \\
-\beta\left[\left(\mathcal{L} \otimes I_{n}\right) \bar{v}_{d}+\operatorname{sig}\left[\left(\mathcal{L} \otimes I_{n}\right) \bar{v}_{d}\right]^{\tau}\right] \\
-\mathfrak{D}\left(M_{d} \otimes I_{n}\right)\left[\operatorname { s i g n } \left[\left(\mathcal{L} \otimes I_{n}\right) \bar{x}_{d}+\operatorname{sign}\left[\left(\mathcal{L} \otimes I_{n}\right) \bar{v}_{d}\right]+\left(M_{d} \otimes I_{n}\right) \Delta .\right.\right.
\end{gathered}
$$

Let $\bar{\zeta}_{d}=\left(\bar{x}_{d}^{T}, \bar{v}_{d}^{T}\right)^{T}$, the system (22) can be written as

$$
\begin{aligned}
& \dot{\bar{\zeta}}_{d}=\left(B_{1} \otimes I_{n}\right) \bar{\zeta}_{d}+\left(B_{2} \otimes I_{n}\right) \operatorname{sig}\left(\overline{\mathcal{L}} \bar{\zeta}_{d}\right)^{\tau} \\
& +\left(-\mathfrak{D} B_{3} \otimes I_{n}\right) \operatorname{sign}\left(\bar{\zeta}_{d}\right)+\left(B_{3} \otimes I_{n}\right) \bar{\Delta} .
\end{aligned}
$$


In what follows, the main results are given in detail.

Theorem 2. Suppose that the directed communication topology is strongly connected. Then, the finite-time consensus for the dynamic system(2) is achieved by the control protocol (5), if $a(\mathcal{L})>\alpha / \beta^{2}, 0<\tau<1$. More specifically, $x_{i} \rightarrow \sum_{j=1}^{N} \xi_{j} x_{j}, v_{i} \rightarrow \sum_{j=1}^{N} \xi_{j} v_{j}, i=1,2, \cdots, N$ in the finite time.

Proof: According to the Lemma 5, zero is a simple eigenvalue of $M_{d}$ with 1 being a corresponding right eigenvector and the other eigenvalues of $\mathcal{L}$ have positive real parts. Then, $\bar{x}_{d}=0$ and $\bar{v}_{d}=0$ if and only if $x_{1}=x_{2}=\cdots=x_{N}$ and $v_{1}=v_{2}=\cdots=v_{N}$, respectively. Therefore, the finite-time consensus problems can be solved if and only if $\bar{x}_{d}$ and $\bar{v}_{d}$ converge to zero in finite time, respectively.

Construct the Lyapunov function,

$$
V_{2}(t)=\frac{1}{2} \bar{\zeta}_{d}^{T}\left(\bar{P}_{1} \otimes I_{n}\right) \bar{\zeta}_{d}
$$

where

$$
\bar{P}_{1}=\left(\begin{array}{cc}
\alpha \beta\left(\Xi \mathcal{L}+\mathcal{L}^{T} \Xi\right) & \alpha \Xi \\
\alpha \Xi & \beta \Xi
\end{array}\right),
$$

According to Lemma 1, we have

$$
\begin{aligned}
V_{2}(t)=\frac{\alpha \beta}{2} \bar{x}_{d}^{T}\left(\Xi \mathcal{L}+\mathcal{L}^{T} \Xi\right) & \left.\otimes I_{n}\right) \bar{x}_{d}+\alpha \bar{x}_{d}^{T}\left(\Xi \otimes I_{n}\right) \bar{v}_{d}+\frac{\beta}{2} \bar{v}_{d}^{T}\left(\Xi \otimes I_{n}\right) \bar{v}_{d} \\
& \geq \frac{1}{2} \bar{\zeta}_{d}^{T}\left(\bar{Q}_{1} \otimes I_{n}\right) \bar{\zeta}_{d}
\end{aligned}
$$

where

$$
\bar{Q}_{1}=\left(\begin{array}{cc}
\alpha \beta a(\mathcal{L}) \Xi & \alpha \Xi \\
\alpha \Xi & \beta \Xi
\end{array}\right) .
$$

According to Lemma $2, \beta>0$ and $a(\mathcal{L})>\alpha / 2 \beta^{2}$, we can get $\bar{Q}>0$, then $V_{2}(t) \geq 0$, and $V_{2}(t)=0$ if and only if $\bar{\zeta}_{d}=\mathbf{0}_{2 N n}$. By using control protocol 
(5), the derivative of $V_{2}(t)$ can be obtained as

$$
\begin{aligned}
& \dot{V}_{2}=\frac{1}{2} \bar{\zeta}_{d}^{T}\left[\left(\bar{P}_{1} B_{1}+B_{1} \bar{P}_{1}\right) \otimes I_{n}\right] \bar{\zeta}_{d}+\frac{1}{2} \bar{\zeta}_{d}^{T}\left[\left(\bar{P}_{1} B_{2}+B_{2} \bar{P}_{1}\right) \otimes I_{n}\right] \operatorname{sig}\left(\overline{\mathcal{L}} \bar{\zeta}_{d}\right)^{\tau} \\
& +\frac{1}{2} \bar{\zeta}_{d}^{T}\left[-\mathfrak{D}\left(\bar{P}_{1} B_{3}+B_{3} \bar{P}_{1}\right) \otimes I_{n}\right] \operatorname{sign}\left(\bar{\zeta}_{d}\right)+\frac{1}{2} \bar{\zeta}_{d}^{T}\left[\left(\bar{P}_{1} B_{3}+B_{3} \bar{P}_{1}\right) \otimes I_{n}\right] \bar{\Delta} \\
& =\frac{1}{2} \bar{\zeta}_{d}^{T}\left[\left(\begin{array}{cc}
-\alpha^{2}\left(\Xi \mathcal{L}+\mathcal{L}^{T} \Xi\right) & 0_{N} \\
0_{N} & -\beta^{2}\left(\Xi \mathcal{L}+\mathcal{L}^{T} \Xi\right)+2 \alpha \Xi
\end{array}\right) \otimes I_{n}\right] \bar{\zeta}_{d} \\
& +\frac{1}{2} \bar{\zeta}_{d}^{T}\left[\left(\begin{array}{cc}
-\alpha^{2} \Xi & -\alpha \beta \Xi \\
-\alpha \beta \Xi & -\beta^{2} \Xi
\end{array}\right) \otimes I_{n}\right] \operatorname{sig}\left(\overline{\mathcal{L}} \bar{\zeta}_{d}\right)^{\tau} \\
& +\frac{1}{2} \bar{\zeta}_{d}^{T}\left[\left(\begin{array}{cc}
-\alpha \mathfrak{D} \Xi M_{d} & -\alpha \mathfrak{D} \Xi M_{d} \\
-\left[\alpha \beta \mathfrak{D} M_{d}(\Xi \mathcal{L}+\mathcal{L} \Xi)+\alpha \mathfrak{D} M_{d} \Xi+\beta \mathfrak{D} \Xi M_{d}\right] & -\left[(\alpha+\beta) \mathfrak{D} M_{d} \Xi+\beta \mathfrak{D} \Xi M_{d}\right]
\end{array}\right)\right. \\
& \left.\otimes I_{n}\right] \operatorname{sign}\left(\bar{\zeta}_{d}\right) \\
& +\frac{1}{2} \bar{\zeta}_{d}^{T}\left[\left(\begin{array}{cc}
\alpha \Xi M_{d} & \alpha \Xi M_{d} \\
\alpha \beta M_{d}(\Xi \mathcal{L}+\mathcal{L} \Xi)+\alpha M_{d} \Xi+\beta \Xi M_{d} & (\alpha+\beta) M_{d} \Xi+\beta \Xi M_{d}
\end{array}\right) \otimes I_{n}\right] \bar{\Delta} \\
& \leq-\bar{\zeta}_{d}^{T}\left(\bar{P}_{1} \otimes \Xi \otimes I_{n}\right) \bar{\zeta}_{d}-\bar{\zeta}_{d}^{T}\left(\bar{P}_{2} \otimes \Xi \otimes I_{n}\right) \overline{\mathcal{L}}^{\tau} \operatorname{sig}\left(\zeta_{d}\right)^{\tau} \\
& -\frac{1}{2} \mathfrak{D} \bar{\zeta}_{d}^{T}\left(\bar{P}_{3} \otimes \Xi \otimes M_{d} \otimes I_{n}\right) \operatorname{sign}\left(\bar{\zeta}_{d}\right)+\frac{1}{2} \bar{\zeta}_{d}^{T}\left(\bar{P}_{3} \otimes \Xi \otimes M_{d} \otimes I_{n}\right) \bar{\Delta} \\
& =-\bar{\zeta}_{d}^{T}\left(\bar{P}_{2} \otimes \Xi \otimes I_{n}\right) \bar{\zeta}_{d}-\bar{\zeta}_{d}^{T}\left(\bar{P}_{3} \otimes \Xi \otimes I_{n}\right) \overline{\mathcal{L}}^{\tau} \operatorname{sig}\left(\bar{\zeta}_{d}\right)^{\tau} \\
& -\frac{1}{2} \bar{\zeta}_{d}^{T}\left(\bar{P}_{4} \otimes \Xi \otimes M_{d} \otimes I_{n}\right)\left[\mathfrak{D} \operatorname{sign}\left(\bar{\zeta}_{d}\right)-\bar{\Delta}\right]
\end{aligned}
$$

Using the assumption that $\left\|\delta_{i}\right\| \leq \mathfrak{D}<\infty$, we have

$$
\dot{V}_{2} \leq-\bar{\zeta}_{d}^{T}\left(\bar{P}_{2} \otimes \Xi \otimes I_{n}\right) \bar{\zeta}_{d}-\bar{\zeta}_{d}^{T}\left(\bar{P}_{3} \otimes \Xi \otimes I_{n}\right) \overline{\mathcal{L}}^{\tau} \operatorname{sig}\left(\bar{\zeta}_{d}\right)^{\tau}
$$

where

$$
\begin{gathered}
\bar{P}_{2}=\left(\begin{array}{cc}
\alpha^{2} a(\mathcal{L}) & 0 \\
0 & \beta^{2} a(\mathcal{L})-\alpha
\end{array}\right), \\
\bar{P}_{3}=\left(\begin{array}{cc}
\frac{\alpha^{2}}{2} & \frac{\alpha \beta}{2} \\
\frac{\alpha \beta}{2} & \frac{\beta^{2}}{2}
\end{array}\right)
\end{gathered}
$$

and

$$
\bar{P}_{4}=\left(\begin{array}{cc}
\alpha & \alpha \\
\alpha \beta+\alpha+\beta & \alpha+2 \beta
\end{array}\right) .
$$


Then, we have

$$
\begin{gathered}
V_{2}(t)=\frac{1}{2} \bar{\zeta}_{d}^{T}\left(\bar{P}_{1} \otimes I_{n}\right) \bar{\zeta}_{d} \\
=\frac{\alpha \beta}{2} \bar{x}_{d}^{T}\left[\left(\Xi \mathcal{L}+\mathcal{L}^{T} \Xi\right) \otimes I_{n}\right] \bar{x}_{d}+\alpha \bar{x}_{d}^{T}\left(\Xi \otimes I_{n}\right) \bar{v}_{d}+\frac{\beta}{2} \bar{v}_{d}^{T}\left(\Xi \otimes I_{n}\right) \bar{v}_{d}, \\
\leq \alpha \beta b(\mathcal{L}) \bar{x}_{d}^{T}\left(\Xi \otimes I_{n}\right) \bar{x}_{d}+\alpha \bar{x}_{d}^{T}\left(\Xi \otimes I_{n}\right) \bar{v}_{d}+\frac{\beta}{2} \bar{v}_{d}^{T}\left(\Xi \otimes I_{n}\right) \bar{v}_{d}, \\
\leq \frac{1}{2} \bar{\zeta}_{d}^{T}\left[\left(\bar{P}_{5} \otimes \Xi\right) \otimes I_{n}\right] \bar{\zeta}_{d},
\end{gathered}
$$

where the positive matrix

$$
\bar{P}_{5}=\left(\begin{array}{cc}
\alpha \beta b(\mathcal{L}) & \frac{\alpha}{2} \\
\frac{\alpha}{2} & \frac{\beta}{2}
\end{array}\right) .
$$

Thus, according to Eq.(29), we can get the following inequality

$$
V_{2}(t) \leq \lambda_{\max }\left(\bar{P}_{5}\right) \xi_{\max } \bar{\zeta}_{d}^{T} \bar{\zeta}_{d}=\bar{m}_{3} \bar{\zeta}_{d}^{T} \bar{\zeta}_{d},
$$

where $\bar{m}_{3}=\left[2 \alpha \beta b(\mathcal{L})+\frac{\sqrt{(2 \alpha \beta b(\mathcal{L})-\beta)^{2}+4 \alpha^{2}}}{4}\right] \xi_{\text {max }}, \xi_{\max }=\max \left\{\xi_{1}, \xi_{2}, \cdots, \xi_{N}\right\}$.

From Eq.(28), we can get the following inequality

$$
\begin{gathered}
\dot{V}_{2}(t) \leq-\lambda_{\min }\left(\bar{P}_{1}\right) \xi_{m i n} \bar{\zeta}_{d}^{T} \bar{\zeta}_{d}-\lambda_{\min }\left(\bar{P}_{2}\right) \xi_{\min } a(\mathcal{L})^{\tau} \bar{\zeta}_{d}^{T} \operatorname{sig}(\bar{\zeta})_{d}^{\tau} \\
=-\bar{m}_{1} \bar{\zeta}_{d}^{T} \bar{\zeta}_{d}-\bar{m}_{2} \bar{\zeta}_{d}^{T} \operatorname{sig}\left(\bar{\zeta}_{d}\right)^{\tau},
\end{gathered}
$$

where $\bar{m}_{1}=\min \left\{\alpha^{2} a(\mathcal{L}) \xi_{\text {min }},\left(\beta^{2} a(\mathcal{L})-\alpha\right) \xi_{\text {min }}\right\}, \bar{m}_{2}=\frac{\alpha^{2}+\beta^{2}}{2} a^{\tau}(\mathcal{L}) \xi_{\text {min }}, \xi_{\text {min }}=$ $\min \left\{\xi_{1}, \xi_{2}, \cdots, \xi_{N}\right\}$. Combining Eq.(30)with Eq.(31), we have

$$
\dot{V}_{2}(t) \leq-\frac{\bar{m}_{1}}{\bar{m}_{3}} V_{2}(t)-\frac{\bar{m}_{2}}{\bar{m}_{3}} V_{2}(t)^{\frac{1+\tau}{2}} .
$$

Based on the above analysis, according to Lemma 3 and inequality (32), we can get the setting time,

$$
T=\frac{2 \bar{m}_{3}}{\bar{m}_{1}(1-\tau)} \ln \frac{\bar{m}_{1} V^{1-\tau}(0)+\bar{m}_{2}}{\bar{m}_{2}} .
$$

Therefore, the finite-time consensus is achieved in system (2) under the directed communication topology. This completes the proof. 


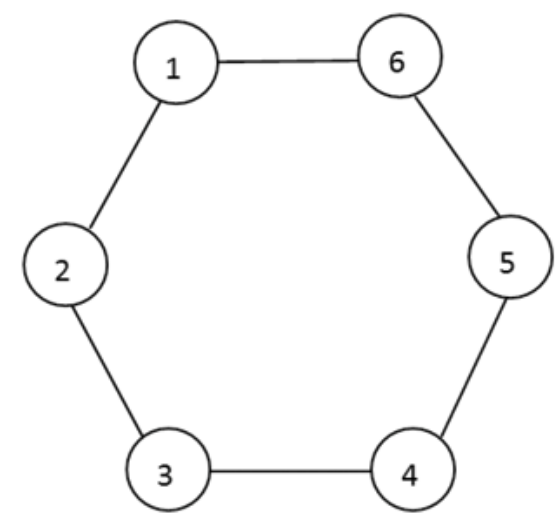

Figure 1: Communication Topology

\section{Numerical Simulation}

Consider multi-agent system (2) of 6 agents with a undirected topology as shown in Fig. 1. The external disturbances are given as $\delta_{i}=0.1 \sin (t)$. Based on Theorem 1, the control parameters in (5) are chosen as $\beta=0.1, \alpha=$ $0.025, \tau=0.2, \mathfrak{D}=0.5$. Fig. $2-6$ show, respectively, position errors, velocity errors, position states, velocity states and control signals of all agents with control protocol as well as their references. It can be seen that the finite-time consensus problem for multi-agent system (2) is achieved less than $t=7 \mathrm{~s}$, which verifies the effectiveness of the theoretical results.

\section{Conclusion}

This paper studied the distributed finite-time consensus control as the multiagent systems with second-order integrators under external bounded disturbances. A distributed finite-time consensus control algorithm has been pro- 


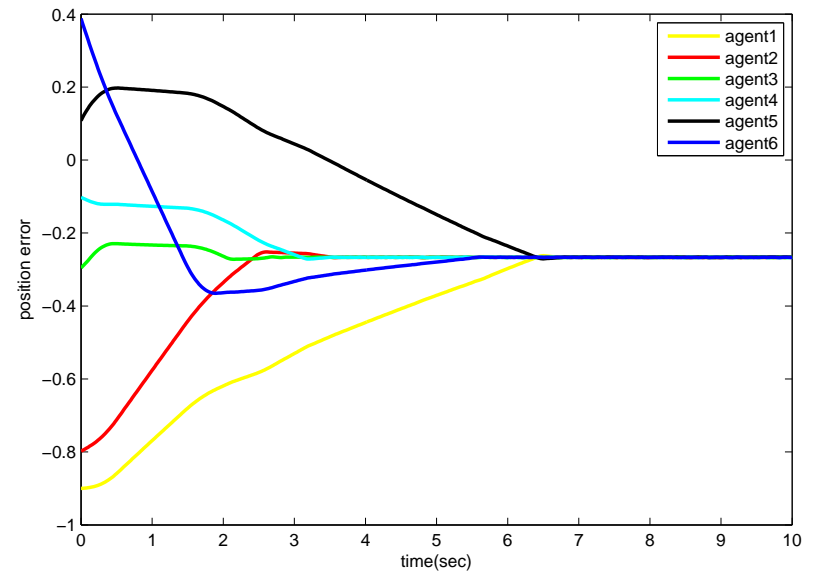

Figure 2: Position errors of all agents

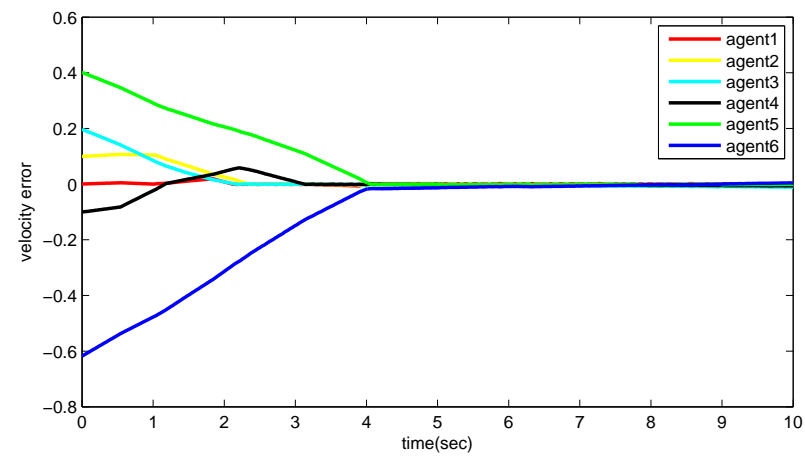

Figure 3: Velocity errors of all agents 


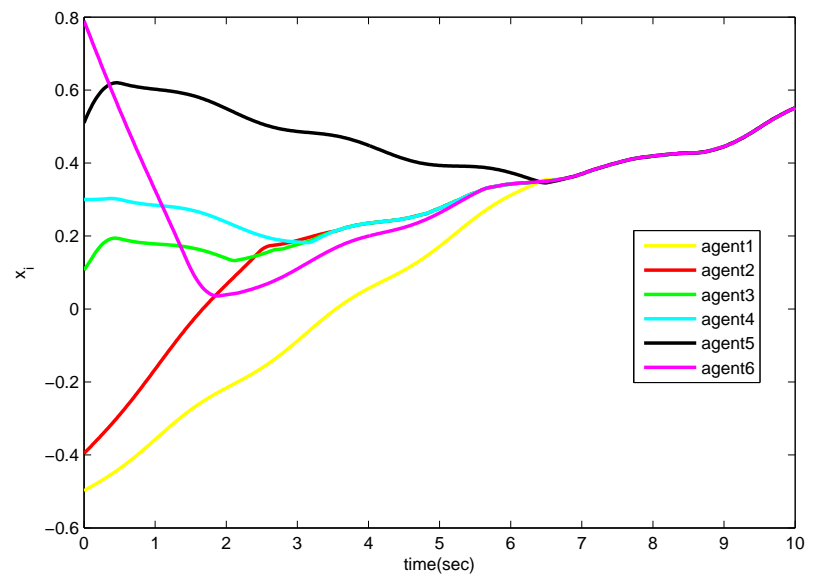

Figure 4: Position states of all agents

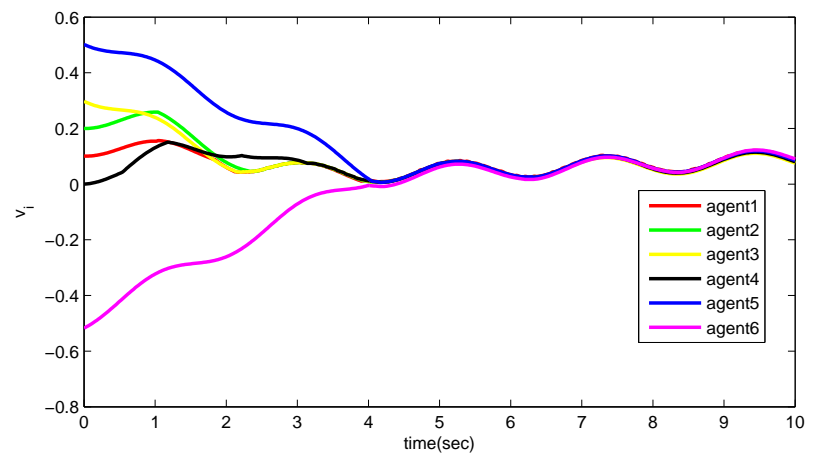

Figure 5: Velocity states of all agents 


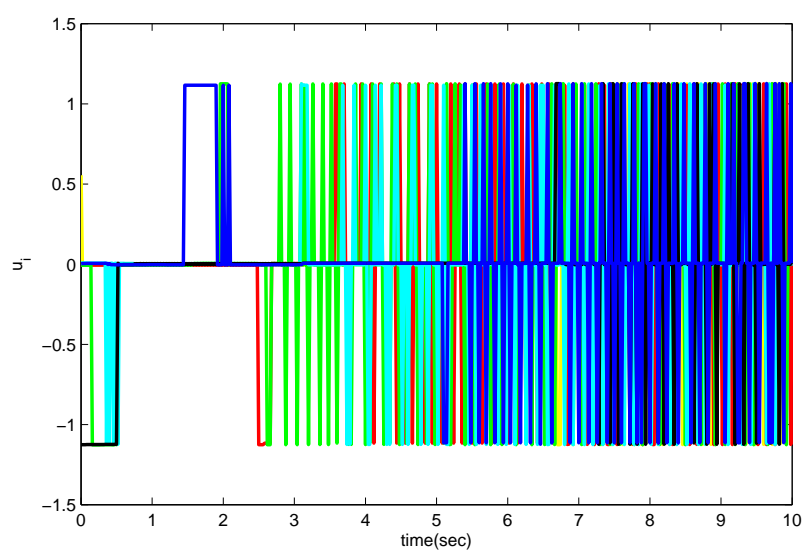

Figure 6: Control signals of all agents

posed based on the relative position and relative velocity information under the undirected and directed communication topologies. By using the Lyapunov stability theorem, matrix theory and graph theory, it is theoretically shown that the states of the agents can be driven to the consensus in the finite time. The simulation results show the effectiveness of the main results.

\section{Acknowledgments}

This research was supported by the National Science Foundation of China (Grants 11602115 and 11572015), the Research Fund for the Doctoral Program of Higher Education (No. 20121102110014), and the Science Research Project for High School of Inner Mongolia (No. NJZY16142).

\section{Reference}

[1] R. O. Saber and R. Murray, Consensus protocols for networks of dynamic agents, Proceedings of the 2003 American Controls Conference. 2003.

[2] H. Wong, M. S. Queiroz, and V. Kapila, Adaptive tracking control using syntheized velocity from attitude measurements, Automatica. 2001, 37(6):947953. 
[3] L. Zhao, C. Hua, Finite-time consensus tracking of second-order multi-agent systems via nonsingular TSM, Nonlinear Dyn. 2014, 75: 311-318.

[4] X. Y. He, Q. Y. Wang, and W. W. Yu, Finite-time distributed cooperative attitude tracking control for multiple rigid spacecraft, Applied Mathematics and Computation. 2015, 256:724-734.

[5] S. H. Li, and Y. P. Tian, Finite-time stability of cascaded time-varying systems, International Journal of Control. 2007, 80(4): 646-657.

[6] Y. Park, Robust and optimal attitude stabilization of spacecraft with external disturbances, Aerospace Science and Technology. 2005, 9(3):253-259.

[7] Y. Zhao, Z. S. Duan, G. H. Wen and G. R. Chen, Distributed $H_{\infty}$ consensus of multi-agent systems: a performance region-based approach, International Journal of Control. 2012, 85(3): 332-341.

[8] B. Zhou, X. F. Liao, Leader-following second-order consensus in multi-agent systems with sampled data via pinning control, Nonlinear Dyn. 2014, 78: $555-569$.

[9] C. Xu, Y. Zheng, H. Su, et al., Cluster consensus for second-order mobile multi-agent systems via distributed adaptive pinning control under directed topology, Nonlinear Dyn. 2016 83: 1975-1985.

[10] W. Ren, R. Beard, E. Atkins, Information consensus in multivehicle cooperative control, IEEE Control Syst. Mag., 2007, 27(20): 71-82.

[11] Z. Y. Meng, W. Ren, Y. C. Cao, and Z. You, Leaderless and leader-following consensus with communication and input delays under a directed network topology, IEEE Transactions on Systems, Man and Cybernetics, Part B: Cybernetics. $2011,41(1)$ : 75-88.

[12] Y. B. Hu, H. S. Su, and J. Lam J, Adaptive consensus with a virtual leader of multiple agents governed by locally Lipschitz nonlinearity, International Journal of Robust and Nonlinear Control. 2013, 23(9): 978-990. 
[13] G. H. Wen, Z. S. Duan, W. W. Yu, and G. R. Chen, Consensus in multiagent systems with communication constraints, International Journal of Roubust and Nonlinear Control. 2012, 22(2): 170-182.

[14] W. W. Yu, W. Ren, W. X. Zheng, G. R. Chen, and J. H. Lü, Distributed control gains design for consensus in multi-agent systems with second-order nonlinear dynamics, Automatica. 2013, 49:2107-2115.

[15] W. W. Yu, G. R. Chen, M. Cao, and J. Kurths, Second-order consensus for multi-agent systems with directed topologies and nonlinear dynamics, IEEE Transactions on Systems. Man, and Cybernetics - part B: Cybernetics. 2010, $40(3): 881-891$.

[16] S. Khoo, L. Xie, Z. Man, Robust finite-time consensus tracking algorithm for multirobot systems, IEEE Transactions. Mechatronics. 2009, 14(2):219228.

[17] Y. C. Cao, and W. Ren, Finite-time consensus for multi-agent networks with unknown inherent nonlinear dynamics, Automatica. 2014, 50(10):26482656 .

[18] M. Ghasemi, and S. G. Nersesov, Finite-time coordination in multiagent systems using sliding mode control approach, Automatica. 2014, 50(4): 12091216.

[19] S. H. Li, H. B. Do, and X. Z. Lin, Finite-time consensus algorithm for multiagent systems with double-integrator dynamics Automatica. 2011, 47:17061712 .

[20] F. L. Sun, and Z. H. Guan, Finite-time consensus for leader-following second-order multi-agent system, International Journal of Systems Science. 2013, 44(4): 727-738.

[21] Y. K. Zhu, X. P. Guan, and X. Y. Luo, Finite-time consensus for multiagent systems via nonlinear control protocols, International Journal of Automation and Computing. 2013, 44(4): 727-738. 
[22] S. H. Li, and X. Y. Wang, Finite-time consensus and collosion avoidnce control algorithms for multiple AUVs, Automatica. 2013, 49:3359-3367.

[23] E. Cruz-Zavala, and J. Moreno, A new class of fast finite-time discontinuous controller, Variable Structure Systems (VSS), 2014 13th International Workshop on. IEEE. 2014: 1-6.

[24] Y. J. Zhang, Y. Yang, Y. Zhao, and G. H. Wen, Distributed finite-time tracking control for nonlinear multi-agent systems subject to external disturbances, International Journal of Control. 2013, 86(1): 29-40.

[25] S. Yu, and X. Long, Finite-time consensus for second-order multi-agent systems with disturbances by integral sliding mode, Automatica. 2015, 54:158165.

[26] X. Y. He, Q. Y. Wang, W. W.Yu, Finite-time containment control for second-order multiagent systems under directed topology. IEEE Transactions. Circuits Systtems. II. 2014, 61(8): 619-623.

[27] X. Y. He, Q. Y. Wang, and W. W. Yu, Distributed finite-time containment control for second-order nonlinear multi-agent systems, Applied Mathematics and Computation. 2015, 268:509-521.

[28] H. B. Do, Y. G. He, and Y. Y. Cheng, Finite-time synchronization of a class of second-order nonlinear multi-agent systems using output feedback control, IEEE Transactions. Circuits Systems. I. 2014, 61(6):1778-1788.

[29] Z. Y. Meng, and Z. L. Lin, On distributed finite-time observer design and finite-time coordinated tracking of multiple double integrator systems via local interactions, International Journal of Roubust and Nonlinear Control. 2014, 24:2473-2489.

[30] Y. Zhao, Z. S. Duan, and G. H. Wen, Finite-time consensus for second-order multi-agent systems with saturated control protocols, IET Control Theory Applications. 2014, 9(3): 312-319. 
[31] R. A. Hong, and C. R. Morse, Topics in matrix analysis, Cambridge, UK: Cambridge University Press. 1991.

[32] S. C. Boyd, L. E. Feron, and V. Balakrishnan, Linear matrix inequalities in system and control theory, Philadelphia, PA:SIAM.

[33] S. P. Bhat, D. S. Bernstein, Finite-time stability of continuous autonomous systems, SIAM Journal. Control and Optimization. 2000, 38(3), 751-766.

[34] W. Ren, and R. W. Beard, Consensus seeking in multiagent systems under dynamically changing interaction topology, IEEE Transactions Automatic Control. 2005, 50(5):655-661. 